\title{
Enhanced Remote Control Providing Medical Functionalities
}

\author{
Adam Bujnowski, Jacek Rumiński, Arkadiusz Paliński, Jerzy Wtrorek \\ Department of Biomedical Engineering \\ Gdansk University of Technology, Gdansk, Poland \\ jwr@biomed.eti.pg.gda.pl
}

\begin{abstract}
This paper presents the enhanced remote control and its role in pervasive healthcare in the home. The device was equipped with health-related measurement modules and a message-processing unit. Preliminary results are presented for monitoring of a pulse, hand tremors, grip forces, and for selfevaluation procedures. The interaction of the device with the smart environment is presented and discussed.
\end{abstract}

Keywords_remote control; pervasive healthcare; assisted living

\section{INTRODUCTION}

Pervasive healthcare environment can provide a model of human-computer interaction in which information processing is integrated into everyday activities for medical diagnostics, therapy and generally improving quality of life. This is especially important given the current trend of an aging population [1][2]. New solutions are required to integrate support for older citizens, their family members and healthcare professionals.

One of the features characterizing older people $(65+)$ is the time spent on watching television. According to recent reports [3] users aged 65 and more spent in the USA almost 48 hours weekly on watching traditional TV. It is about 7 hours a day, and almost two times more than for young users (aged 18-24). Therefore, it is potentially useful to adapt domestic TV infrastructure for improving the quality of life of an elderly person at home.

A Remote Control (RC) is now a very popular tool for management of household audiovisual equipment including TV sets. It is a very useful device that supports the elderly (particularly those with limited mobility), and usually it is located in the immediate vicinity of the person. Therefore, the remote controller may be used as a device allowing collection of information on its user. Given the growing number of health disorders [4] linked to population ageing (e.g. disorders of the cardiovascular system, the Parkinson's syndrome, the risk of stroke, etc.), some additional, health-related functionalities can be added to the RC. Portable devices, including mobile phones, have been proposed to provide additional functions for ambient assisted living [5], supporting health monitoring [6], therapy [7], etc. Additionally, it has been proposed to use handhelds for wireless control of different PCs and appliances [8][9]. In [10], a special language is proposed for describing the functionality of appliances and to automatically generate both graphical and

This work has been partially supported by European Regional Development Fund concerning the project: UDA- POIG.01.03.01-22-139/0900 "Home assistance for elders and disabled DOMESTIC", Innovative Economy 2007- 2013, National Cohesion Strategy. speech user interfaces on handheld computers.

Biomedical signals have been used for wireless control of devices. For example, in [11] the author proposed a heart rate sensing device that relays heart rate to a remote control unit for controlling entertainment devices. In [12] authors proposed a remote control for television comprising a skin spectroscopy sensor able to measure the characteristics of the skin, and means to send a signal allowing identifying the particular user currently using the remote control.

In this short paper we present methods and solutions to enhance a remote control with medical diagnostics functionalities and for processing of messages in pervasive healthcare in the home. The functionalities of the RC were discussed with the group of psychologists and professional caregivers from local Municipal Social Welfare Centers. The group of experienced professionals (16), representing more than 500 caregivers, was selected by managers of municipal centers. Discussions took place during a specially organized workshop in December 2012. However, in this paper we focused only on the $\mathrm{RC}$ as a device to collect vital signs seamlessly and to get immediate feedback from the assisted person. The presented interfaces have been design to validate the proof of the concept. The next steps of the research will focus on the design of the user interfaces and studies with cooperation of elderly people from the centers we work with.

\section{METHODS}

An experimental method was used to investigate the possibility of expansion of a remote control for monitoring of selected medical parameters and to support user interaction in the home.

\section{A. The enhanced remote control}

A remote control can be enhanced with embedded medical diagnostic sensors to monitor vital signs and other healthrelated parameters. Vital signs include the heart beat, breathing rate, temperature, and blood pressure [13]. Additionally, oxygen saturation in blood $(\mathrm{SpO} 2)$ is often included in the group of the most important vital signs [14].

The following modules have been designed for the enhanced remote control: 1-lead ECG module, pulse (SpO2) oximeter, a grip force meter, a hand-tremor meter with accelerometer, a vibrator, a set of LEDs (a small flashlight), a loudspeaker, a microphone, a LCD display, and a module for 
wireless communication. The simplified architecture of the designed remote control called Human Assistance Remote Navigator (HARN) is presented in fig. 1 .

All modules have been embedded in the portable remote control (fig.2), which has been also designed as universal, traditional remote control for TV sets and appliances.

Two microcontrollers (MCU) were used. The first microcontroller controls dedicated modules, the keyboard, the display, infrared transmission circuit, etc. The second one, the signal microcontroller, is devoted to process voice signal and to manage communication links. It can be used for processing signals obtained through the microphone and recognition of particular words (voice commands) spelled by the user. This is additional data entry interface. The microcontrollers communicate with each other via $\mathrm{I} 2 \mathrm{C}$ bus.

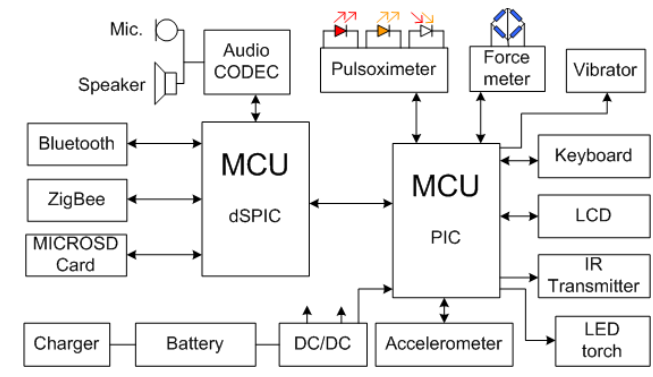

Fig. 1. The simplified block diagram of the HARN remote control

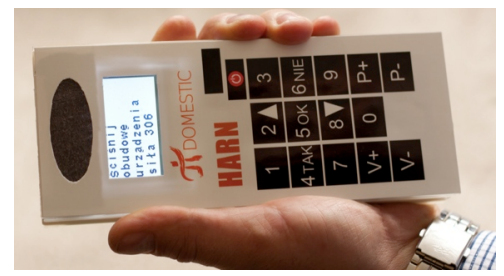

Fig. 2. The first prototype of the HARN remote control

The ECG electrodes and force transducers are embedded in the sidewalls of the unit. The $\mathrm{SpO} 2$ reflective optical sensor is located in the front side, below the display. The RC can work in different operation modes selected from a menu presented on the display by means of navigation keys: a "classical" $\mathrm{RC}$, force meter, $\mathrm{SpO} 2$ meter, accelerations meter, etc. Special procedures have been implemented to use signals from the accelerometer to create a tilt mouse (a HID device).

\section{B. The pervasive healthcare environment}

In the pervasive healthcare environment, especially in the context of ambient assisted living, information processing is integrated into everyday objects and activities. In the smart homes a network of sensors and diagnostic devices (e.g. weighing scales, blood pressure devices, etc.) is connected to a central, computer station (fig.3) [15]. The station collects, processes, and presents information for selected users (e.g. an assisted person, a family member, a nurse, etc.).

The HARN has been designed to play different roles in such an environment. First, it is used to collect information in a form of vital signs and parameters representing the current status of the person. The central computer can process received information to detect unusual events and values or to analyze long-term trends. Currently, the HARN provides: 1-lead ECG signal, SpO2 parameter value, a pulse value, symmetry of grip forces in both hands, frequency of hand tremor, and amplitudes of tremor. The last parameters are collected automatically (i.e. without special attention of the user) whenever the remote controller is used. This allows collecting data in long term and monitoring trends in values.

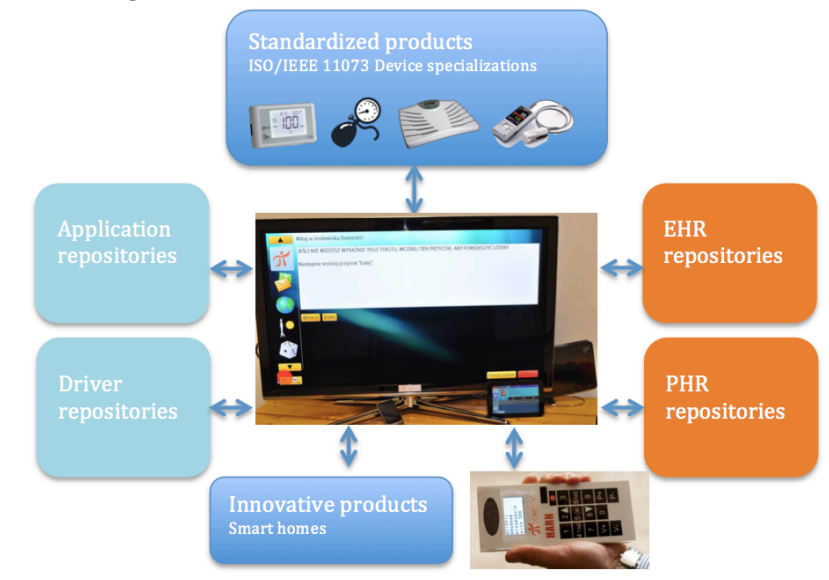

Fig. 3. The pervasive healthcare environment at home. The central workstation (micro computer) plays a role of a hub for sensors and devices.

Additionally, the embedded message-processing unit provides means to analyze status of the assisted person (e.g. "How are you today?", "The same?", "Worse than yesterday?"). It is also possible to collect information about responses of the user to the events, e.g. "Did you take medication?". The messages, questions, etc., can be transmitted to the remote control using the central station as a middleware. For example, a nurse or a family member can configure synchronous messages for the assisted person or she or he can invoke messages asynchronously (e.g. using the authorized smartphone outside the home of the assisted person).

The central station (e.g. set-top box) can be connected to a TV set, where information is presented to the assisted user. The HARN enables the control of the graphical user interface of the central station using voice commands, the keyboard, or a tilt mouse (based on processing of data from accelerometers). Additionally, the remote control has been designed for processing of alerts. It can be both source of an alert message (e.g. using a virtual "HELP ME" button) or a receiver (observer) of alerts. In the last role the assisted user can interact with the alert (e.g. confirm it).

\section{RESULTS}

In this short paper preliminary results are presented based on implemented HARN remote control and the smart home environment.

The pulse value was measured for seven healthy volunteers (age: $34 \pm 13.1$ ) simultaneously using the HARN (fig. 4) and the certified medical device. There were no important differences observed (i.e. grater than $6 \%$, assumed acceptable for screening purposes). The frequency and the amplitude of hand tremor was measured using laboratory stand and from 
the assisted person. The laboratory setup used a generator of periodical signals (with controlled frequencies in the range 3$10 \mathrm{~Hz}$ ) and a speaker (source of tremors). The Parkinsonian Tremor (PT) was measured for a user in rest condition. Results obtained for both configurations proved the possibility of quantitative evaluation of tremor frequency. In fig. 5 examples of original PT signal from accelerometer (in time domain) and power spectrum with detected PT frequency $(5.66 \mathrm{~Hz})$ are presented. The remote control (using an accelerometer) is very suitable for such measurements, because the direction of hand tremor has important vertical character.
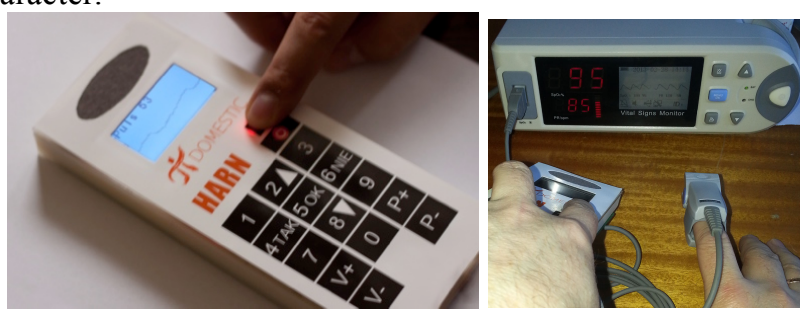

Fig. 4. Examples of measurement of a pulse using optical sensor
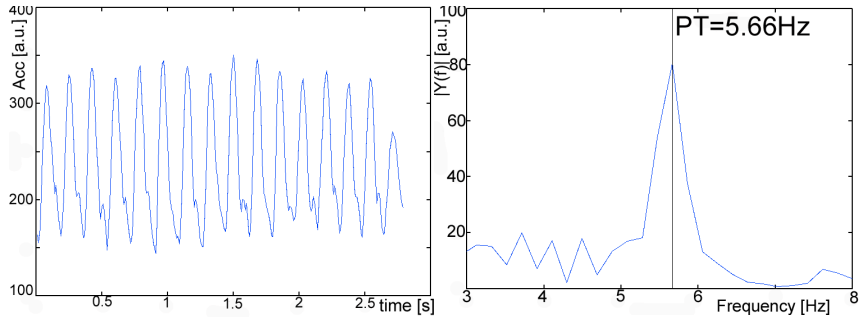

Fig. 5. Examples of real data acquired for rest Parkinsonian Tremor (PT) using the HARN remote control: signal from accelerometer in time (left), power spectrum with detected PT frequency, $5.66 \mathrm{~Hz}$ (right)

Figure 6 shows an example of grip force recorded during 11s. In addition, stability is shown in the form of grip forces trend.

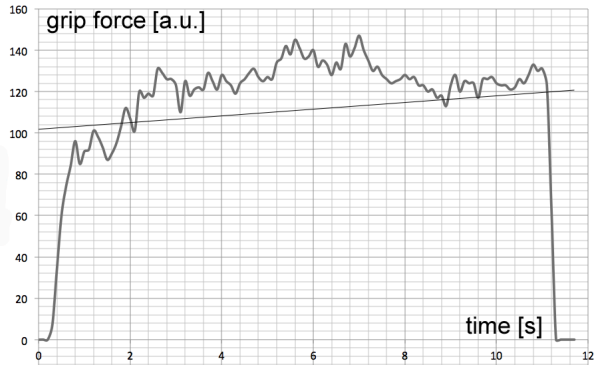

Fig. 6. Example of a grip force signal measured with the HARN RC

The procedure implemented in the HARN RC guides the user to measure the grip force sequentially in both hands. The symmetry of grip force (max values and trends) is an important indicator of motor asymmetries, usually resulting from serious health incidents (e.g., a stroke). The HARN RC is equipped with various means of providing information to the user: the LCD display, the vibrator, the speaker, the set of LEDs, and wireless communication modules (infrared and Bluetooth). The communication modules enable communication with a TV set, computers, or with other sensor nodes in the pervasive environment. In fig. 7 examples of possible messages presented on the LCD display are shown. The presented images illustrates only the proof of the concept and are not a real messages designed for the end-user. The format and content of the message (configurable on the device) can be adjusted depending on the user perception (size and color of the text, voice messages, vibrations, etc.) and requirements. This can be developed according to the idea proposed in the Global Public Inclusive Infrastructure (GPII.NET).
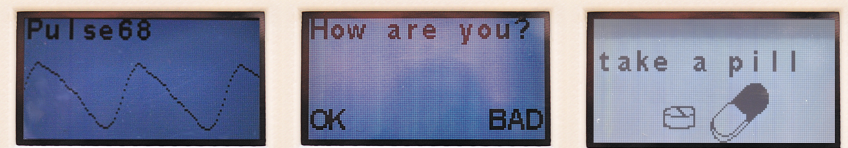

Fig. 7. Examples of messages presented on the display of the HARN RC

A message may be generated by a sensor (e.g. a water leakage sensor), the user's family member, the user, by the central station, etc. The central station processes all messages and distributes them to predefined receivers. For example, the home drug dispenser sends a message that the user did not take his medication. The central station transmits the alert (or question) to the RC, to other devices (e.g. TV) and to other system users (e.g. a nurse). The alert is presented as a voice message and is displayed on the RC (with light and sound signalization) waiting for the user action. Other examples related to interaction using messages can be represented by configurable questions like: "Did you measure your blood glucose today?“, „Need grocery shopping?“, etc. In the implemented HARN RC it is possible to introduce practically every message text (with a given character length limit). If the message is in the form of a question then it is mapped to a function in a programming language, which returns an integer code (int question(char * questionText)). The return code is mapped to a value in accordance with a specific vocabulary (e.g., "How are you feeling today?" 0 - "OK", 1 - "THE SAME AS YESTERDAY", 2 - "WORSE THAN YESTERDAY, "How strong do you feel pain (0-9: 0-no pain; 9-worst pain)?").

Figure 8 presents examples of messages generated by the central station using measurements/events from the HARN remote control: on a mobile phone (left) and on a desktop screen (right). For example, reduced daily activity can be observed when the assisted person does not use the $\mathrm{RC}$ in the previously observed way. The "if-then" rules are used.

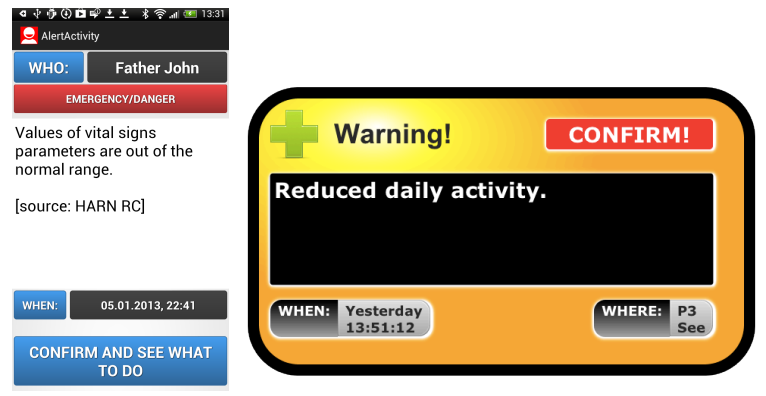

Fig. 8. Examples of alert messages generated by the central station using measurements/events from the HARN remote control: on a mobile phone (left), on a desktop screen (right) 


\section{DISCUSSION AND CONCLUSION}

Seniors will accept a new hardware interface much easier if it is similar to the device that they have already used successfully [16]. The remote controllers utilized for controlling household appliances can be relatively easily equipped with additional functionalities supporting assisted living. The implemented functionalities in the presented $\mathrm{RC}$ were discussed with formal and experienced caregivers. It can be further expanded with other sensors (e.g. temperature sensor, glucometer) and used for different medical problems (transient ischemic attack). However, the most important feature of the device is the possibility of medical data measurement and transmission to the central station. Regularly collected data are processed for long-term estimation of user behavior (e.g. daily activity) and time-related health concerns. This is a unique feature of application of the RC for long-term medical analysis. Another an important feature of the proposed interface is its ability to extension for new sensors and message distribution models. In this paper a single user of the remote controller was assumed. Introducing simple identification methods like dedicated manipulators, RFID tags, etc., can support multiple users.

Preliminary results indicate that it is possible to use the enhanced remote controller in the pervasive healthcare environment. In addition to the typical measurement modules (e.g. 1-lead ECG, SpO2) particular role plays units designed for the measurements of frequencies and amplitudes of hand tremors and the symmetry of grip force. In the longitudinal study of tremor frequencies in Parkinson's disease and essential tremor it was proved that accelerometers can provide valuable information about tremor frequencies and tremor frequency decrease with time [17]. Using the HARN RC it is possible to introduce every day measurements in the nondisturbing way. Health care providers can evaluate results of such measurements to evaluate when the tremor appears, when it changes its character (frequencies), etc. Similarly, measurement of grip force can be especially important for long-term analysis or as a symptom of dangerous incidents (e.g. stroke leads to motor asymmetries). Bimanual asymmetries in stroke survivors indicate the need for identifying and treating the task-specific impairments for maximizing motor recovery [18]. Other tests are possible using the HARN RC, for example registering whistling (with the microphone), evaluation of hand movements, etc. The HARN $\mathrm{RC}$ can be used to detect selected symptoms of dangerous incidents and can be used in therapy following the incident.

The phrase "fifth vital sign" is usually used to indicate pain, as perceived by the patient (using a scale of 0-10) [19]. The HARN RC can be used in the subjective assessment of the level of pain or change in pain (e.g. in time). Similarly other self-evaluations are possible.

In this short paper limited number of measurements were presented. However, most of the measurement methods are based on the well-know procedures (e.g. using accelerometers for tremor analysis). The originality of this paper is the introduction of integrated measurements in the device interacting within the pervasive environment. Further research is required to design user interfaces for the target group and to perform studies with end-users. Remote controls are well-know devices, accepted by most users. The elderly use a remote control often, so it is usually located close to the assisted person. Therefore, the enhanced remote control can play an important role in monitoring of selected health-related parameters and can provide important information about the status of the person in non-disturbing way.

\section{REFERENCES}

[1] Administration on Aging, A Profile of Older Americans: 2011, U.S. Department of Health \& Human Services, 2011

[2] European Union, The 2012 Ageing Report: Underlying Assumptions and Projection Methodologies. EUROPEAN ECONOMY 4, European Union, 2012

[3] Nielsen Company, State of the media: The cross-platform report, www.nielsen.com, USA, 2012.

[4] World Health Organization ,Global status report on noncommunicable diseases 2010, Description of the global burden of NCDs, their risk factors and determinants, World Health Organization, pp: 1-176, April 2011.

[5] Doughty K., "SPAs (smart phone applications) - a new form of assistive technology", Journal of Assistive Technologies, Vol. 5 Iss: 2, pp.88 94, 2011

[6] Bolkhovsky J. B., Scully C. G., Chon K. H., Statistical Analysis of Heart Rate and Heart Rate Variability Monitoring Through the Use of Smart Phone Cameras, 34th Annual International Conference of the IEEE EMBS San Diego, California USA, pp.1610-1613, 2012.

[7] Helal S., Giraldo C, Kaddoura Y., Lee Ch., El Zabadani H., Mann W., "Smart Phone Based Cognitive Assistant". The 2nd International Workshop on Ubiquitous Computing for Pervasive Healthcare Applications (UbiHealth2003), October 12, 2003.

[8] Myers B. A., Using handhelds for wireless remote control of PCs and appliances. Interacting with Computers, 17(3):251-264, 2005.

[9] Nilsson T., New Remote Control Service on Xperia ${ }^{\mathrm{TM}}$ smartphones, Sony Mobile Developer World, http://developer.sonymobile.com/, 2012.

[10] Nichols J., Myers B. A., "Creating a Lightweight User Interface Description Language: An Overview and Analysis of the Personal Universal Controller Project". ACM Transactions on Computer-Human Interaction, Vol. 16, no. 4, (November 2009). pp. 1-37., 2009.

[11] Volpe J.C, Heart rate sensor for controlling entertainment devices, US Patent 6572511, USA, 2003.

[12] Dagaeff T., Fullton L., Christophe Oddou, Remote control with sensor, EP 2429183, 2012.

[13] Dugdale D.C., Vital Signs, MedlinePlus Encyclopedia, http://www.nlm.nih.gov/medlineplus/ency/article/002341.htm, 2013.

[14] Mower W, Myers G, Nicklin E, Kearin K, Baraff L, Sachs C (1998). "Pulse oximetry as a fifth vital sign in emergency geriatric assessment". Acad Emerg Med 5 (9): 858-65.

[15] Wtorek J., Bujnowski A., Ruminski J., Polinski A. Kaczmarek M., Nowakowski A., Assessment of cardiovascular risk in assisted living, Metrology and Measurement Systems, 19(2), pp. 231-244, 2012.

[16] Bures V., Interactive Digital Television and Voice Interaction: Experimantal Evaluation and Subjective Perception by Elderly, Electronics and Electrincal Engineering, 2012, 6(122):87-90.

[17] Hellwig B, Mund P, Schelter B, Guschlbauer B, Timmer J, Lücking $\mathrm{CH}$., A longitudinal study of tremor frequencies in Parkinson's disease and essential tremor., Clin Neurophysiol. 2009 Feb;120(2):431-5.

[18] Lodha N, Patten C, Coombes SA, Cauraugh JH., Bimanual force control strategies in chronic stroke: finger extension versus power grip, Neuropsychologia, 50(11):2536-45, 2012.

[19] Department of Veteran Affairs, Pain as the 5th vital sign toolkit, Geriatrics and Extended Care Strategic Healthcare Group, Veterans Health Administration, Washington, 2000. 